könnte uns also leicht etwas geben, was für viele andere zu schwer wäre. Er hat offenbar absichtlich den rein praktischen Standpunkt so vorangestellt. Darum ist das Glossar mit grausamer Strenge auf eine Weise angeordnet, welche zwingt, erst einige grammatische Elemente sich anzueignen. Bisher hat man diese bescheidene Forderung möglichst vermieden. Hoffen wir auf jeden Fall, dass St. den libyschen Studien treu bleibt, trotz der Kleinheit des zu erwartenden Leserkreises. Er verspricht Sprachproben aus dem tunesischen Gebiet; aus seinen kabylischen Zitaten schliesse ich, dass er uns auch eine neue, erschöpfende Grammatik dieses Dialektes liefern könnte, was keineswegs überflüssig wäre. Die Peinlichkeit des Herausgebers ist sogar in dem äusserst sorgfältigen Druck (berichtige noch S. 12, Z. 9, 10; 114,5ff.) erkennbar. Schade, dass ich den Wert für die Kenntnis des marokkanischen Arabisch nicht zu beurteilen vermag!

Philadelphia.

Mahler Ede, Az Egyiptomi nyelo alapelemei. Budapest 1899. 4. 21 u. $90 \mathrm{~S}$. Bespr. v. A. Wiedemann. Vorliegende Schrift von Eduard Mahler ist, so viel ich sehe, die erste Grammatik des Altaegyptischen, welche in ungarischer Sprache erscheint. So erfreulich es auch ist, dass derart die Kenntnis der Aegyptologie in immer weitere Kreise dringt, so wird das Buch nur auf einen kleinen Leserkreis rechnen können, da die Kenntnis des Ungarischen nicht zum Rüstzeuge des Orientalisten zu gehören pflegt. Wir begnügen uns daher auch an dieser Stelle mit einer kurzen Inhaltsangabe. Der Beginn ist in Typen gesetzt; er enthält nach einem Vorwort allgemeine Bemerkungen über die aegyptische Sprache, die Schrift, die Entzifferung, die Litteratur und ein leider durch zahlreiche Druckfehler entstelltes Verzeichnis einer Reibe moderner aegyptologischer Bücher und Aufsätze. Dann folgen autographiert eine kurze Grammatik, deren Angaben wesentlich auf Erman und daneben auf Brugsch beruhen, mit beigefügten Beispielen, eine kleine Sammlung von Lesestücken, einige Sylbenzeichen mit ihren Lesungen und ein Glossar.

Bonn.

(dem z. B. noch urti-hortas zuzaweisen wäre). Immerhin liesse sich wohl Stoff zu einer kleinen Monographie sammeln.

\section{Die Schlussworte des demotischen Papyrus Insingor.}

Von Wilhelm Spiegelberg.

Der jetzt im Leidener Museum befindliche Papyrus, welcher soeben in einer mustergültigen Weise ron Pleyte und Boeser veröffentlicht worden ist, enthält 4 Schlusszeilen, deren Sinn nicht ohne weiteres zu Tage liegt. Zunächst die Uebersetzung, welche sich von derjenigen der Herausgeber nur in einigen Stücken entfernt "Der Schluss des Königlichen Buches. Es verjüngt sich seine Seele in alle Ewigkeit! - Der Ibis, um den Affen (d. i. Thot) zu erheitern. Für seinen (sc. des Ibis) Geist, um dem Osiris-Sokaris, dem grossen Gott, dem Herrn von Abydos zu dienen. Es verjüngt sich seine Seele auf seinem Leibe bis in alle Ewigkeit". Im Gegensatz zu den Herausgebern fasse ich er $\mathrm{t}^{\mathrm{i}}$-hra deshalb nicht als Imperativ, weil sich die sonst häufige Imperativform mit / in unserem Texte nicht nachweisen lässt. Ferner nehme ich $\underline{t}^{i}-h^{2}{ }^{2}$ in dem gut belegten Sinn des Prototypes sd;(i⿱亠) hr $h$ erheitern", aus welchem sich im kopt. die Bedeutung njocari ${ }^{*}$ entwickelt hat ${ }^{1}$ ).

Was ist nun aber dieser dunklen Rede Sinn? - Die erste Zeile, für welche wir kurz "finis" setzen würden, hat nichts mit den letzten beiden zu thun, die eine in sich geschlossene eigenartige Formel enthalten. Diese ist uns aus Mumienetiketts bekannt. Was nämlich auf „der Ibis, um den Affen zu erheitern" folgt, ist die Formel ${ }^{2}$ ), welche sich in so vielen Mumientiketts an den Namen des Toten anschliesst. Folglich muss der "Ibis 4 dem Toten ${ }^{3}$ ) entsprechen.

Wenn man sich nun vergegenwärtigt, dass häufig bei Tiermumien - so noch jüngst bei den Ausgrabungen von Grenfell und Hunt im Fajum - Papyrusrollen gefunden warden, so hat man des Rätsels Lösung. Der grosse Leidener Papyrus war einer Ibismumie beigegeben worden, welche ihrerseits eben durch ihre Beigabe den affengestaltigen Gott Thot, dessen heiliges Tier der Ibis ist, "erheitern" sollte. Da Thot der Gott der Wissenschaft ist, so wählte man einen litterarischen Text, eben das vorliegende „könig-

1) Max Müller: Die Liebespoesie der alten Aegypter, S. 39 Anm. 9.

2) Für diese Formel verweise ich auf den im Drack befindlichen ersten Band der "demotischen Studien".

3) Die Anschauung, den verstorbenen Ibis ganz wie einen verstorbenen Menschen zu betrachten, liegt z. B. auch in der Bezeichnung "Osiris Ibis, der selige" vor (Verzeichnis der agypt. Altertümer des Berliner Museums, 2. Auflage, 8. 310). 
liche Buch", an dessen "Lehren" der Gott seine besondere Freude haben musste. Dafür sollte er dann dem verstorbenen Ibis die Seligkeit verschaffen, welche in der Umgebung des Osiris zu finden war.

\section{Die Suklim.}

Von W. Max Müller

C. Niebuhr's Mitteilung OLZ. III, 69 enthält eine glänzende Entdeckung in der Beobachtung, dass das Afrikanervolk der Q 2 Chr. 12,3 dasselbe ist wie das Produkt des Landes Ophir, die תוכיים die angeblichen „Pfauen ${ }^{\text {1) }}$. Doch bürdet Niebuhr denen, welche sich mit Mizraim und Kusch beschäftigt haben, noch immer die verzweifelte Aufgabe auf, ein solches Volk nachzuweisen, wie die alten Ửbersetzungen ("Trog(l)odyten") es wollen. Allein von einem solchen Stamm ist keine Spur zu finden; er bleibt nebelhaft.

Ich habe schon Asien, S. 111 Anm. die "Pfauen" angezweifelt, aber nicht den Mut gehabt, die "Emendation des schweren Wortes", die sich mir aufdrängte, in den Text zu setzen. Die Hauptfrage ist: gehört das Wort zu der guten alten Überlieferung oder zu den späteren Erweiterungen? In den Text hat man ja auch das Silber hineingeflickt, das ein Kenner der Verhältnisse so wenig aus dem Roten Meer kommen lassen konnte, wie wir Orangen aus Grönland. Das Wort חביים fehlt beidemal im Vaticanus. Doch bleibt die Möglichkeit, dass die ältere LXX es als unverständlich ausliess (?), noch offen. Ist es aber ächt, so möchte ich lesen : *an [nächste Stufe der Verderbnis * * daher das "], d. h. das ägyptische Wort tsm „Windhund ${ }^{\varkappa 2}$ ). Vgl. zu der häufigen Erwähnung der schnellen Jagdhunde aus Nubien und Punt, Asien 117, (im einzelnen: Petersburger Papyrus; Hoskins, Travels, Tf. zu 321, - wo zu Koš gezogen -, Ros. Civ. 17,7, DHI II, 12) die nach WZKM. $X, 209$ nicht libyschen, sondern kuschitischen Namen von König Antef's Leibhunden. Die Schreibung סכיים weist vielleicht noch darauf hin, dass ursprünglich ein $\mathbf{s}$ in dem Wort stak, doch kann das s auch sekundär sein.

1) Das Hübscheste ist, dass die alten Rabbinerschulen nach der Punktation das auch schon verglichen haben müssen.

2) Das Wort bedeutet in Ägypten speziell den Windhand, wird aber auch auf andere Jagdhunde ubertragen. Die Hunde von Punt sind schwerer gebaut und gohorren einur eigentümlichen Rasse an, welche zu untersuchen sich vielleicht für Kenner der Zoologie lohnte.
Ich glaube, diese Emendation ist leichter als die umgekehrte Annahme, סביים habe irgendwie "schwarze Menschen" geheissen. Die Sklaven stehen bei der ägyptischen Aufzählung der „Wunder von Punt" zuletzt, weil sie das für die Ägypter am wenigsten Fremdartige waren; für die Hebräer wäre das bei Negern weniger einleuchtend. - Freilich, wie das Wort zu einem Völkernamen wurde, das auszudenken, erforderte einige Phantasie. Irgendwie muss der Text doch einmal in der einen oder anderen Stelle bedeutend verändert worden sein. Das zu untersuchen, überlasse ich anderen.

Ich wiederhole also: den von Niebuhr entdeckten Zusammenhang der zwei Wörter ' $n$ und ' $D$ glaube ich so zu begreifen, dass $\cap$ das Ursprüngliche ist und ' $D$ daraus $a b$ geleitet. Wenigstens solange das letztere Wort so ganz rätselhaft bleibt, ziehe ich das vor.

\section{Über eine vierte Kopie der grossen Karnakliste. \\ Von W. Max Müller.}

Im März 1896 schrieb mir Prof. A. H. Sayce von Siut einige freundliche Mitteilungen über neue Funde in Aegypten und erwähnte darin die Entdeckung einer neuen Kopie der grossen Liste palästinischer Städte sus der Zeit Dhutmose III. Ich machte davon keinen Gebrauch, da ich voraussetzte, dieser Fund würde bald ausführlich veröffentlicht werden. Nachdem aber mehrere Jahre vertlossen sind, scheint es mir rätlich, jene Notiz abzudrucken, um auf die Notwendigkeit einer Herausgabe des ganzen Textes hinzuweisen. Auch angenommen, dass er gar nichts besonders Neues enthielte, so wäre er zur Kontrolle der drei bekannten Kopien und zur Beurteilung ihres Verhältnisses von Nutzen. Es wird so viel gleichgiltiges Zeug abgedruckt, aus dem man schwerlich je etwas Nützliches ausziehen wird, warum muss ein historisches Denkmal verfallen, ohne dass man sich seiner erbarmt?

Sayce schrieb: The excavations in the great Fore-Court (A in Baedeker's plan) have brought to light, at the foot of the column, immediately to the east of the chapel of Seti II, the angle of a wall composed of stone taken from a new copy of the Palestine list of Thotmes III. Nos. $1-8,9$ 14, 49-51, $53-66,76-85$ are represented, tho'many of the names are effaced. The only $\nabla a$ riants in it which $I$ have found are: 